\title{
Diagnóstico do serviço de abastecimento de água na percepção do usuário no município de Barcarena - Pará
}

\author{
Diagnosis Of Water Supply System Based On The User Perception in the City of Barcarena (Pará - Brazil) \\ Keyt Oliveira Gonçalves'; Lindemberg Lima Fernandes² e Luiza Girard ${ }^{3}$ \\ 1,2,3 Universidade Federal do Pará
}

\begin{abstract}
Resumo
Este trabalho teve como objetivo diagnosticar o serviço de abastecimento de água do bairro Centro no município de Barcarena-Pará, através da percepção do usuário. A metodologia utilizada foi a aplicação de questionários, os quais foram tratados através de método estatístico, sendo aplicados em 166 residências nos anos de 2013 e 2014. De acordo com os resultados, verificou-se que grande parcela dos entrevistados (66\%) não tem acesso ou não utiliza o serviço público de abastecimento de água, sendo os poços individuais a solução utilizada. A pesquisa revelou ainda que fatores como interrupção no fornecimento (69\%) e deficiência na qualidade da água (90\%) podem ser responsáveis pela busca de outras fontes de abastecimento. $\mathrm{Na}$ opinião dos usuários, a água apresenta características indesejáveis, tais como cor e sabor, fator este que faz com que o consumo de água envasada seja elevado no local, chegando a $58 \%$ das residências. Portanto, a metodologia de avaliação do sistema a partir da percepção do usuário mostrou-se ferramenta importante para a gestão, para o planejamento e para a credibilidade do sistema.
\end{abstract}

Palavras-chaves: : Sistema de abastecimento de água. Usuário. Diagnóstico.

\begin{abstract}
This study aimed to diagnose the water supply system of the district of Barcarena-Pará, through the user's perception. Questionnaires were applied in 166 homes in the years 2013 and 2014. The data collected were treated by statistical methods. According to the results it was found that a large proportion of the sample $(66 \%)$ do not have access or does not use the public service of water supply and individual wells is the solution used. The survey also revealed that factors such as disruption in the water supply system (69\%) and inadequate water quality (90\%) may be responsible for seeking alternative sources of supply. In the view of the users water presents undesirable characteristics such as color and flavor and this factor causes the consumption of bottled water, reaching $58 \%$ of the sample. Therefore, the methodology for evaluating the system from the user's perception was an important tool for managing, planning and system credibility.
\end{abstract}

Keywords: Water supply system. User. Diagnosis. 


\section{INTRODUÇÃO}

Uma preocupação da população tem sido o acesso à água potável, em quantidade e qualidade, devido a questões de escassez e deterioração das águas.

Como uma grande parte da população do mundo já vem, atualmente, enfrentando problemas de escassez de água, os serviços de água urbanos enfrentam desafios substanciais para a gestão dos recursos hídricos (RISCH et al., 2014).

A falta de acesso à água potável vem sendo apontada como a causa da maioria das enfermidades em países em desenvolvimento. Atualmente, a preocupação com a proteção de áreas destinadas ao abastecimento público é eminente, pois a disponibilidade de água é cada vez menor. Entre as prováveis fontes de poluição das águas estão os aterros sanitários mal operados, os aterros controlados e lixões (ROWE; CARDOSO, 2009) e o lançamento de efluentes.

Segundo IBGE (2010), o número de domicílios atendidos por rede de abastecimento de água no Brasil passou de $78 \%$ para $83 \%$. Apesar de verificado um crescimento quanto ao atendimento no fornecimento de água tratada no país, a avaliação da eficácia desse serviço torna-se necessária para possíveis adequações.

Estudos de mananciais, distribuição e consumo de água têm um papel importante no planejamento municipal dos sistemas (NNAJI et al., 2013). Neste sentido, uma das ferramentas é a avaliação do sistema de abastecimento a partir da percepção do usuário, pois assim é possível demonstrar a sua satisfação de forma que venha a subsidiar os gestores na tomada de decisões voltadas à melhoria continua e sustentável do sistema.

De acordo com Nascimento et al. (2013) apud Tuan (2012), a percepção é resposta dos sentidos aos estímulos ambientais, e a atividade mental, resultante da relação com o meio ambiente, proporcionando ao indivíduo a compreensão de seu entorno, estabelecendo relações com o ambiente no qual está inserido.

No entanto, estudos que investigam aspectos sociais para analisar a aceitação pública e as suas preocupações ainda são escassos (MAINALI et al, 2013). Assim, torna-se necessário conhecer as dinâmicas dos processos de consumo de água nas localidades a fim de gerar processos de gestão desde este nível até níveis superiores (SILVA et al.,2012).

Sendo assim, este trabalho visa diagnosticar a prestação do serviço de abastecimento de água no bairro Centro da cidade de Barcarena-Pará a partir da percepção do usuário quanto ao uso da água.

\section{METODOLOGIA}

O estudo foi desenvolvido no município de Barcarena no estado do Pará, no bairro centro, conforme Figura 01, o qual apresenta cerca de 1700 imóveis.

$\mathrm{O}$ município pertence à mesorregião Metropolitana de Belém e a sede municipal apresenta as coordenadas geográficas de $01^{\circ} 30^{\prime} 24^{\prime \prime}$ de latitude ao Sul e 4837'12" de longitude a Oeste de Greenwich. Seus limites estão na Baía do Guajará com o município de Belém ao Norte, nos municípios de Mojú e Abaetetuba ao Sul, a Leste com a Baía do Guajará e o município de Acará, e a Oeste com a Baía do Marajó. Segundo IBGE (2010), apresentava uma população de 94.641 habitantes.

O estudo foi realizado em duas etapas, em que a primeira constou de realização de revisões bibliográficas para a fundamentação teórica e a caracterização do sistema de abastecimento de água, bem como para a identificação das características do local de estudo. A segunda etapa referiu-se às atividades de campo, onde foram aplicados questionários contendo perguntas objetivas.

A técnica de amostragem da população entrevistada foi definida através do uso de um método estatístico, o qual, segundo Oliveira (2004), se leva em consideração o nível de confiança, o erro amostral o qual se pretende admitir e a proporção amostral, bem como o tamanho da população. Seu dimensionamento foi feito para população finita, sendo o valor obtido para a amostra de 166 residências, conforme a Equação 01. 


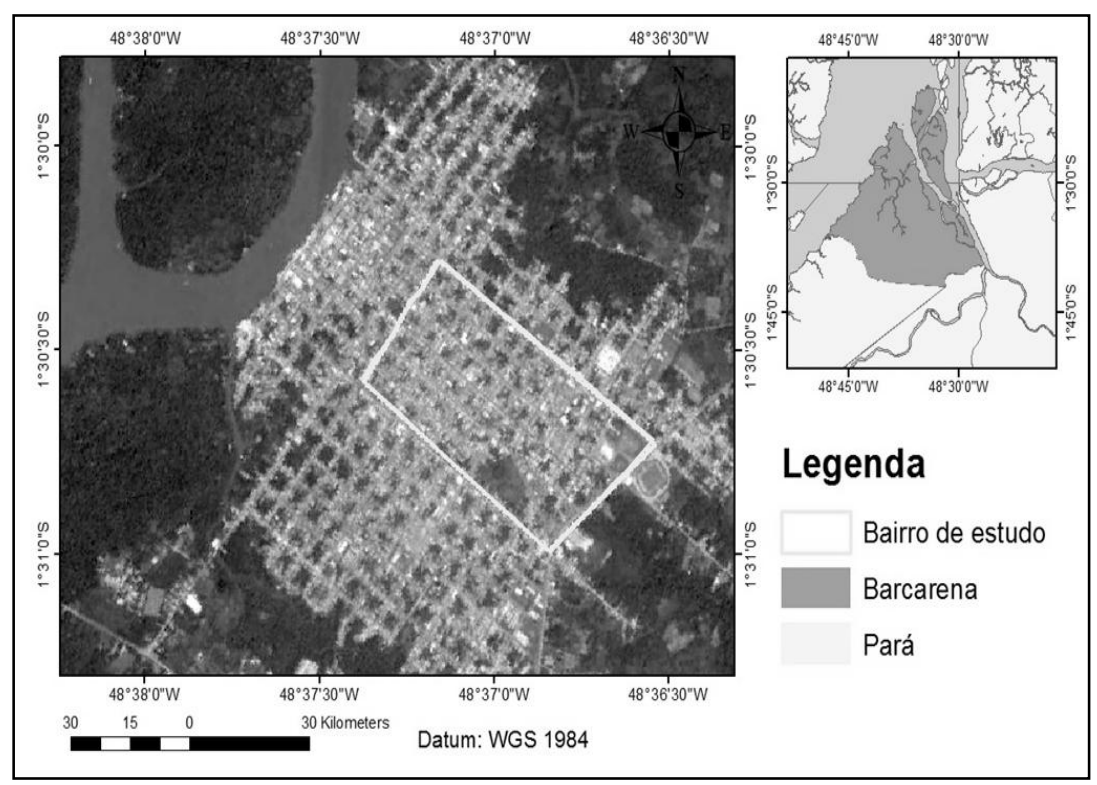

Figura 01 - Área de estudo - Bairro Centro

Fonte: Adaptado Google Earth, 2014

$$
n=\frac{Z^{2} \cdot N \cdot(p \cdot q)}{Z^{2}(p \cdot q)+(N-1) \varepsilon^{2}}
$$

Onde:

$\mathrm{Z}=$ abscissa da curva normal padrão, fixado nível de confiança de $97 \%=2,17$

$\mathrm{N}=$ tamanho populacional $=1700$

$\varepsilon=$ erro amostral $=8 \%$

$\mathrm{p} \times \mathrm{q}=$ proporção amostral $=0,25$.

Para análise dos dados, foram utilizados o Microsoft Excel e o Access 2007, onde foram sistematizados e tratados os dados dos questionários e apresentados seus respectivos resultados em forma de gráficos.

\section{RESULTADOS}

$\mathrm{Na}$ análise dos dados foi identificado que a maioria dos consumidores no bairro é de categoria doméstica (88\%), o que demonstra que, apesar de o bairro ser definido como o centro da cidade, a atividade comercial se mostra em pouca proporção no local.

Foi detectado que apenas uma pequena parcela das residências do bairro em estudo é atendida pelo serviço de abastecimento público de água. Isto se deve ao fato de o reservatório elevado estar desativado, sendo assim, o sistema não dispõe de pressão suficiente para fornecer água para as residências mais distantes, levando os usuários a buscarem outras formas de abastecimento, como o uso de poços particulares (66\%). Assim, a cobertura do sistema público de abastecimento de água alcança apenas $(31 \%)$ dos imóveis amostrados.

No que concerne ao fornecimento de água, Figura 03 , foi verificado que apenas em $6 \%$ das residências amostradas não ocorre interrupção no fornecimento de água, enquanto na maioria (60\%) falta água periodicamente, o que reforça o quanto é fundamental a existência de um reservatório, pois, como o mesmo está desativado, a água é bombeada diretamente para as residências e, nos momentos de falta de energia, o fornecimento de água é paralisado. 


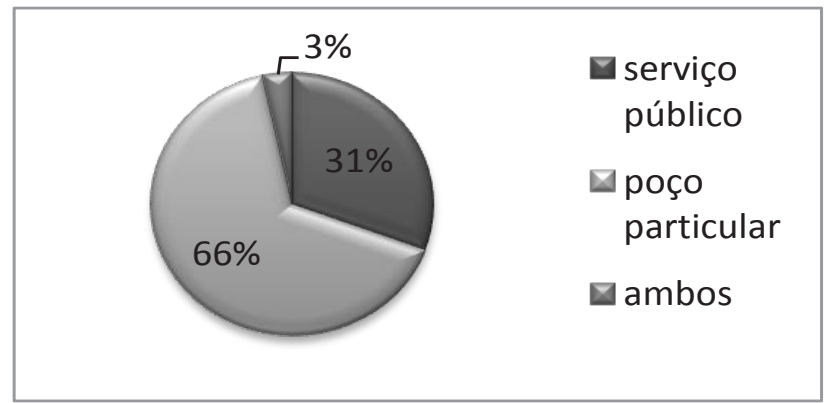

Figura 02- Fonte de abastecimento dos imóveis

Fonte: autor, 2014

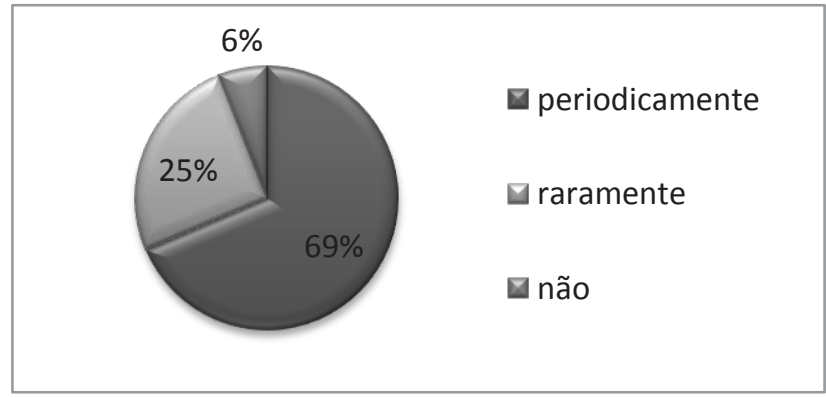

Figura 03 - Interrupção no fornecimento de água do sistema público.

Fonte: autor, 2014

Quanto à existência de medidor de água, no local da pesquisa (bairro Centro) foi verificado que não há hidrometração do sistema. Assim, foi questionado se os entrevistados concordavam ou não com a hidrometração das residências. Do total da amostra, $61 \%$ disse concordar com a hidrometração, entretanto, quando perguntado aos que aceitaram com a micromedição de sua residência, cerca de $71 \%$ respondeu não concordar, conforme Figura 04.

Os entrevistados ressaltaram os motivos para a recusa em aceitar a instalação de hidrômetros em suas residências: controle do volume utilizado (tarifa) e qualidade da água. Em relação a este último, 90\% disse que a água possui cor e sabor, o que, na percepção dos usuários, são características desagradáveis em uma água para consumo.

Além desses motivos, foi mencionada também a questão de que a água deveria ser de graça

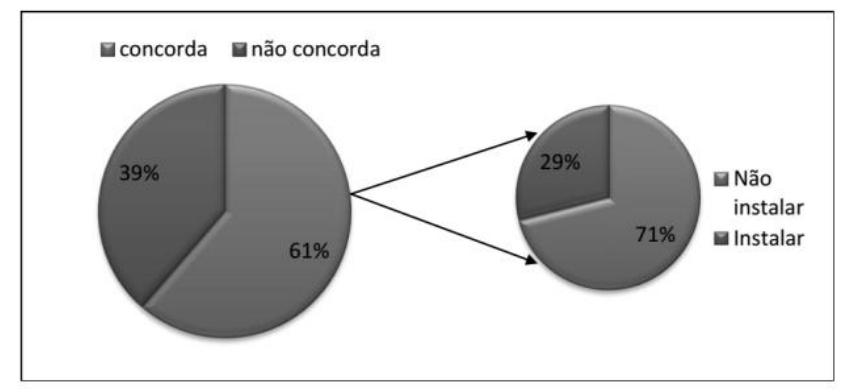

Figura 04 - Interrupção no fornecimento de água do sistema público

Fonte: autor, 2014

para todos, sem nenhum tipo de cobrança quanto ao seu uso, já que é um recurso natural. Com isso, pode-se observar que o usuário desconhece o que ele realmente paga, que neste caso não é a água e sim o serviço de fornecer a água tratada e canalizada à população. 
Quanto ao consumo de água para ingestão, de acordo com a Figura 05 , verificou-se que $58 \%$ dos entrevistados disse consumir água envasada, o restante utiliza a filtração da água do sistema ou de poço.

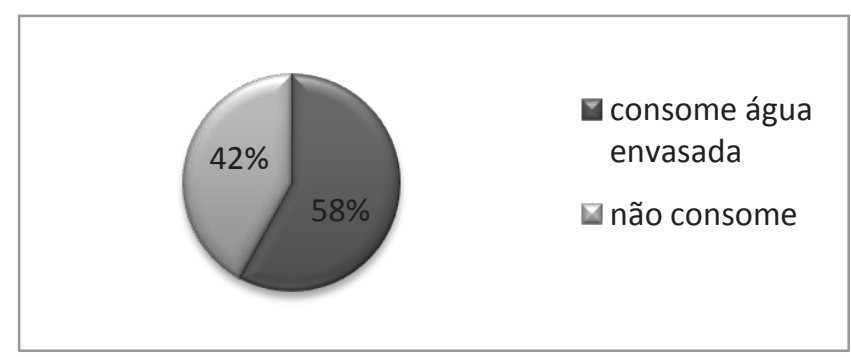

Figura 05 - consumo de água envasada

\section{CONSIDERAÇÕES FINAIS}

De acordo com os resultados obtidos, verificou-se que a gestão do serviço público de abastecimento de água, no bairro Centro do município de Barcarena não é adequada, pois, através da percepção dos usuários, foram identificadas falhas no sistema, destacando-se que $66 \%$ dos entrevistados disse consumir água de poço particular para suprir a falta de acesso à água da concessionária local. Outro indicativo de falha foi o relato de interrupção no fornecimento de água. Além disso, tem-se a insatisfação da população quanto às características da água.

Apesar de $61 \%$ dos entrevistados concordar com a instalação de hidrômetros, destes, $71 \%$ disse não aceitar a instalação em sua residência, o que pode estar relacionado tanto à fragilidade do sistema quanto ao aumento da fatura de água.

Portanto, a metodologia de avaliação do sistema a partir da percepção do usuário, mostrouse ferramenta importante, que em conjunto com as ações de operação, manutenção e melhorias e expansão dos serviços por parte da concessionária, são imprescindíveis à gestão e ao planejamento e à credibilidade do sistema.

\section{REFERÊNCIAS}

INSTITUTO BRASILEIRO DE GEOGRAFIA E ESTATÍSTICA (IBGE). Pesquisa Nacional de Saneamento Básico - Rio de Janeiro, 2010.

MAINALI, Bandita; PHAM, Thi Thu Nga; NGO, Huu Hao; GUO, Wenshan; MIECHEL, Clayton; O'HALLORAN, Kelly; MUTHUKARUPPAN, Muthu; LISTOWSKI, Adnrzej. Vision and perception of community on the use of recycled water forhousehold laundry: A case study in Australia. Science of the Total Environment, 463-464:657-66, 2013.

NASCIMENTO, Ruth Silveira do; RIBEIRO, Maria Adriana de Freitas Mágero; BARBOSA, Dayse Luna; OLIVEIRA, Rui; MEIRA, Celeide Maria Belmont Sabino; OLIVEIRA, Lorena Thaís Freitas; LUCENA, Dátia Paula Marques Maia. Análise da percepção sobre a qualidade do sistema de abastecimento de água na cidade de Campina Grande - Paraíba. In: XX Simpósio Brasileiro de Recursos Hídricos. ABRH. Bento Gonçalves-RS, 2013.

NNAJI, Chidozie Charles; ELUWA, Chinedum; NWOJI, Chioma. Dynamics of domestic water supply and consumption in a semi-urban Nigerian city. Habitat International, 40, p 127-135, 2013.

OLIVEIRA, Paulo Henrique F.C. Amostragem básica - Aplicação em auditoria. Ed. Ciência Moderna Ltda, 2004. 
RISCH, Eva; LOUBET, Philippe; NÚÑEZ, Montserrat; ROUX, Philippe. How environmentally significant is water consumption during wastewater treatment?: Application of recent developments in LCA to WWT technologies used at 3 contrasted geographical locations. Water Research, Vol 57, pp 20-30, 2014.

ROWE, Elmo J.; CARDOSO, Décio L. A utilização de mapas temáticos no diagnóstico de áreas suscetíveis à contaminação por percolado de aterro sanitário. Eng. Agrícola, Jaboticabal, v.29, n.4, p.670-678, 2009.

SILVA, Deibys Gildardo Manco; ERAZO, Jhoniers Guerrero; CRUZ, Ana María Ocampo. Eficiencia en el consumo de agua de uso residencial. Revista Ingenierías Universidad de Medellín, vol. 11, No. 21 pp. 23-38, 2012. 